\title{
Redesign of a supply network by considering stochastic demand
}

\author{
Juan Camilo Paz, Julián Andrés Orozco, Jaime Mauricio Salinas, Nicolás Clavijo Buriticá and John \\ Willmer Escobar*
}

Department of Civil and Industrial Engineering, Pontificia Universidad Javeriana Cali, Colombia

\begin{tabular}{|c|c|}
\hline$\overline{C H R O N ~ I ~ C ~ L ~ E ~}$ & AB S T R A C T \\
\hline $\begin{array}{l}\text { Article history: } \\
\text { Received January } 162015 \\
\text { Received in Revised Format } \\
\text { April } 102015 \\
\text { Accepted April } 162015 \\
\text { Available online } \\
\text { May } 22015 \\
\text { Keywords: } \\
\text { Supply Network Design } \\
\text { Logistics } \\
\text { Variability of the Demand } \\
\text { Sample Average Approximation } \\
\text { Stochastic Linear Programming }\end{array}$ & $\begin{array}{l}\text { This paper presents the problem of redesigning a supply network of large scale by considering } \\
\text { variability of the demand. The central problematic takes root in determining strategic decisions } \\
\text { of closing and adjusting of capacity of some network echelons and the tactical decisions } \\
\text { concerning to the distribution channels used for transporting products. We have formulated a } \\
\text { deterministic Mixed Integer Linear Programming Model (MILP) and a stochastic MILP model } \\
\text { (SMILP) whose objective functions are the maximization of the EBITDA (Earnings before } \\
\text { Interest, Taxes, Depreciation and Amortization). The decisions of Network Design on stochastic } \\
\text { model as capacities, number of warehouses in operation, material and product flows between } \\
\text { echelons, are determined in a single stage by defining an objective function that penalizes } \\
\text { unsatisfied demand and surplus of demand due to demand changes. The solution strategy adopted } \\
\text { for the stochastic model is a scheme denominated as Sample Average Approximation (SAA). } \\
\text { The model is based on the case of a Colombian company dedicated to production and marketing } \\
\text { of foodstuffs and supplies for the bakery industry. The results show that the proposed } \\
\text { methodology was a solid reference for decision support regarding to the supply networks } \\
\text { redesign by considering the expected economic contribution of products and variability of the } \\
\text { demand. }\end{array}$ \\
\hline
\end{tabular}

\section{Introduction}

A supply network is a set of coordinated echelons that supply the demand of products ordered by the customers. A suitable network design must seek simultaneously to reduce operational costs and maximize profits, while that tends to increase or maintain an adequate service level. Building a network design that supports decision making considering these two objectives simultaneously can be a difficult task for most organizations (Chen et al., 2005). Additionally, distribution networks typically operate under an uncertain environment, so it has become even more important for companies to maintain a robust supply network design (Melo et al., 2009).

A supply network design (SND) implies typically the decision making process at strategic and tactical levels. The strategic level decisions consider different aspects associated with determining the location 
and the level of capability of any echelon of the network. These decisions include irreversible capital allocations and are inked to the overall profitability and other key performance measures such as lead time, inventory level, responsiveness to the variability of demand, quality, among other.

This paper, addresses the supply network redesign problem for consumer products companies, is dedicated to the production and marketing of foodstuffs and supplies for the bakery industry. The proposed models have been tested with real data from the most representative Colombian company in this sector. The company network is composed by 6 factories, which produce semi-finished and finished products. The semi-finished products are used as inputs for manufacturing other products. The network must supply 15 commercial districts from its warehouses and distributions centers (DC).

First, a deterministic Mixed Integer Linear Programming model (MILP) is formulated, representing the network complexity. The model considers the average demands of products in each market area and the maximization of profits before tax and amortization (EBITDA) as the objective function. In addition, a Stochastic Mixed Integer Linear Programming Model (SMIPL) has been formulated. The decisions of the stochastic model are determined in a single step by penalizing unsatisfied demand and surplus of demand generated after variability of demand. It is proposed as real objective function of the SMIPL model (penalizing unsatisfied demand and surplus), the maximization of profits before tax and amortization, the latter is defined as the difference between expected revenues and costs associated with decisions made by the model. The solution strategy adopted for the SMILP model solution is known as Sample Average Approximation (SAA). This methodology has been developed by Kleywegt et al. (2002) and uses a scheme of sample averages by Monte Carlo Simulation for stochastic linear programming problems.

The main contribution of this paper is the mathematical structure of the proposed SMILP model. This structure allows determining strategic and tactical decisions from the supply networks stochastic optimization with the SAA algorithm in a single step. In the literature reviewed, all previous works that use SAA as solution method also use a two-stage scheme for the SND problem. In addition, the paper extends the literature of mathematical modeling applied to SND by considering variability of demand and maximizing the expected revenues by sales. In particular, we seek to evaluate the applicability and effectiveness of a stochastic linear model for strategic and tactical decision making in redesigning large scale supply chain.

In Section 2, the literature review on supply chain design with stochastic elements is presented. In Section 3 , the experimental methodology proposed for the problem development is presented. Finally, computational results and conclusions are presented in Section 4 and 5 respectively.

\section{Literature review}

The research in the field of designing distribution networks dates back to theories developed in the early nineteenth century proposals primarily by agricultural, economist and geographers (Ballou, 2004). The common theme of all these works, was the recognition of transportation costs to determine the best location of facilities; concepts that are applied in the current theory. Many experts have significantly contributed to the development of the evolution of the network design theory (Berman \& Drezner, 2003; Current et al., 1998) and different objective functions have been formulated for numerous applications, simultaneously (Beamon, 1999, Owen \& Daskin, 1998). Owen and Daskin (1998) mentioned the importance of SND problem in strategic planning for companies of national and international operations and its impact on the success or failure of customer satisfaction.

Two types of efficiency measures have been used predominantly in the supply network design: minimizing logistics costs and maximizing demand compliance level. Arabani and Farahani (2012) performed an extensive review of different types of optimization objectives set for SND models. 
According to Arabani and Farahani (2012), in most papers related to these models, the typical objective functions are minimizing: cost, response time, distance and risk associated with the design of the supply chain; and an extent profit maximization is rarely considered. This can be explained because profit maximization can cause not all demands are supplied if the associated costs are greater than the income. Among the few studies that consider profit maximization, we found approaches related to the maximization of the difference between revenues and costs as well as the maximization of the profit after tax. Meixell and Gargeya (2005) presented a review of the work related to supply chain design and defined different aspects considered such as tax rate, exchange rate, lead time, etc.

Problems of design of multiservice networks with multiple layers have been considered by Klose and Drexl (2005). In this paper, different types of specific logistic distribution networks to meet a certain kind of demand were considered. In addition, there have been recent efforts to incorporate into the design of these chains, the reverse logistics; in some cases this may be a crucial factor for SND because it could adversely affect the objective function. This work has not considered these processes since the redesign was performed on a chain with mass consumption products, where the product value is not sufficiently high or representative against the cost of integrating it back to the chain.

The proposed research is related to the consideration of stochastic components within SND models. In this topic, researchers such as Owen and Daskin (1998) provided an overview of this type of modeling, considering the stochastic nature of the parameters used in the supply chain design, allowing models much more adjusted to the actual operating conditions. Some robust models of probabilistic networks design by considering the widest possible set of random parameters have been studied by Chen et al. (2005), Gabor and Van Ommeren (2006), Escobar (2009) and Escobar et al. (2012, 2013), etc. On the other hand, most of SND models under uncertainty consider the minimization cost or the maximization of the average revenue expected (Snyder, 2006; Petrovich, et al., 2008). In particular, the study of variability in SND has been divided into three broad categories: scenario-based approximation, probabilistic approximation and stochastic programming (Escobar et al., 2012; Escobar, 2012). Stochastic programming considers the optimization of a problem with uncertain parameters having or not a known probability distribution. Several works that consider SND by using stochastic programming have been proposed by Chen et al. (2003), Santoso et al. (2005), Listes and Dekker (2005), Snyder (2006), Gabor and Van Ommeren (2006), Lieckens and Vandaele (2007), Coronado-Hernández et al. (2010) and Escobar et al. (2012). This type of work uses the two-stage stochastic problem given by Dantzing (1955). In this work, the first step seeks to minimize the sum of the first stage costs, which are known; while the second stage seeks the minimization of the expected cost of flow variables in the network problem.

This paper proposes the development of a single-stage stochastic programming model considering the variability of demand for the SND problem. The proposed method is based on the strategy algorithm SAA (Kleywegt et al., 2002). First, a limited sample of supply network configurations is generated; in which each of these is determined from multiple random generation of multiple random demand scenarios and corresponding stochastic model solutions. From this sample the indicators proposed by Kleywegt et al. (2002) are calculated to verify the stopping criterion, then the process is repeated until the criterion is met, ensuring the selection of the best configuration. Some works of network models that consider SAA as solution strategy for design of large-scale real networks are developed by Chouinard et al. (2008) and Schutz et al. (2009).

\section{Experimental methodology}

\subsection{Supply Network Design: Deterministic Model (MILP)}

In this section, the mathematical model proposed for the redesign of the supply network is described. In particular, for the MILP we have considered the average demand for the product $g$ at market area $z$. 
- We have considered distribution process of several echelons by modeling the flow of products between groups of plants, from plants to distribution centers, between distribution centers, from distribution centers to commercial districts, from distribution centers to market areas (direct delivery) among commercial districts, and from commercial districts to market areas.

- The manufacturing plants send groups of products to other plants, which serve as input for the production of other products (semi-finished products).

- All the physical network infrastructures are assumed within a single country (Colombia) excluding exports. The buying process of groups of imported products and domestic products is performed at the distribution centers and warehouses.

- The models are designed to consider a single period planning (1 year) and consider 38 groups of products, in which 32 correspond to finished products and 6 semi-finished products that serve as input for manufacturing some finished products. The group of finished products includes 400 items, which have been categorized according with their common characteristics. The flow through the network is considered at tons transported in each groups of items between locations.

- Because the distribution centers are close to the plants, the transportation costs between these facilities are considered negligible.

- We consider an initial infrastructure of plants, distribution centers and warehouses already established seeking to review closure of warehouses. We do not consider closing or opening of plants or distribution centers.

- Production and storage capacity constraints are considered at plants, warehouses and distribution centers. The production and storage capacity is determined in relation to the tons of groups of items flowing through the network. We allow extra production capacity by penalizing it.

- Transport capacity limitations between plants and distribution centers are not considered. The selected mode of transportation is truck (decisions of transport modes selection are not included).

- The setup costs of groups of items in plants and distribution centers are considered including loading cost.

- The freight charges from the commercial districts to the market areas are considered as weighted values. In particular, they are estimated as average value paid by tons of group of items sent to the market areas.

- We consider average weighted sales prices for each group of items in each market area.

- Two types of customers are considered: customers attended by direct delivery from distribution centers and customers attended by delivery from warehouses.

- The network supplies to two types of demands, the demand generated in the market areas and the demand generated by direct dispatches; the latter is associated with a specific group of customers.

The objective function of the mathematical model for the supply network seeks to maximize the profits before tax and amortization (EBITDA) by considering decisions related to close warehouse commercial districts and increased production capacity.

\subsection{Notation of Deterministic Model MILP}

Sets

$P L \quad$ Set of manufacturing plants, indexed by $i$,

$C D \quad$ Set of distribution centers, indexed by $j$,

$A \quad$ Set of warehouses, indexed by $k$,

$Z \quad$ Set of market areas or customers, indexed by $z$,

$G A \quad$ Set of groups of items, indexed by $g$,

GAS Set of raw materials groups and semi-finished articles indexed by $e$. 
Subsets

$G A C O N \subseteq G A$

$G A C O I \subseteq G A$

$G A 2 \subseteq G A$

$G A P_{i \in P L} \subseteq G A$

$P L P_{g \in G A} \subseteq P L$

$C D G A_{g \in G A} \subseteq C D$

$P L C D_{j \in C D} \subseteq P L$

$G A S$

$G A S G A_{g \in G A 2} \subseteq$

$G A$

$P L G A S_{e \in G A S} \subseteq$ $G A$

Variables

$P_{e i q g}$
$B_{g i j}$
$Y_{g j n}$
$D_{g j k}$
$E_{g k l}$
$F_{g k z}$
$H_{g j z}$
$W_{k}$
capdil $_{i g}$
capdil $_{i e}$

Parameters

$P V_{g z}$

$C C P_{g}$

$C P_{g i}$

$C P G A S_{e i}$
Set of group of items selling in the national market $g \in G A$,

Set of group of imported items $g \in G A$,

Set of group of items $g \in G A$ which require semi-finished products $e \in G A S$ to be produced,

Set of group of items $g \in G A$, that can be produced in the plant $i \in P L$, Set of plants $i \in P L$ which can produce the articles group $g \in G A$,

Set of distribution centers $j \in C D$ which can dispatch the group of items $g \in G A$, Set of plants $i \in P L$ which can dispatch to the distribution center $j \in C D$,

Set of raw materials groups and semi-finished articles indexed by $e$.

Set of semi-finished group of items $e \in G A S$ used to manufacture the group of items $g \in G A 2$,

Set of plants $i \in P L$ which can manufacture the group of semi-finished items $e \in$ GAS.

Quantity of group of items semi-finished $e \in G A S$ to be sent from the plant $i \in P L$ to the plant $q \in P L$ to produce the group of items $g \in G A, i \neq q$ [weight unit /time unit],

Quantity of group of items $g \in G A$ to be sent from the plant $i \in P L$ to the distribution center $j \in C D$ [weight unit/time unit],

Quantity of group of items $g \in G A$ to be sent from distribution center $j \in C D$ to the distribution center $n \in C D, j \neq n$ [weight unit/time unit],

Quantity of group of items $g \in G A$ to be sent from the distribution $j \in C D$ to the warehouse $k \in A$ [weight unit/time unit],

Quantity of group of items $g \in G A$ to be sent from the warehouse $k \in A$ to the warehouse $l \in A, k \neq l$ [weight unit/time unit],

Quantity of group of items $g \in G A$ to be sent from the warehouse $k \in A$ to the market area $z \in Z$ [weight unit/time unit],

Quantity of group of items $g \in G A$ to be sent from the distribution center $j \in C D$ to the market area $z \in Z$ [weight unit/time unit],

Binary variable associated to the warehouse $k \in A$ : " 1 " if decide to close, " 0 " otherwise,

Additional production capacity for the plant $i \in P L$ for group of items $g \in G A$ [weight unit/time unit],

Additional production capacity for the plant $i \in P L$ for the materials or semi-

finished product $e \in G A S$ [weight unit/time unit].

Average sales price of the group of items $g \in G A$ in the market area $\mathrm{z} \in \mathrm{Z}$ [\$/weight unit],

Average buying cost of group of items $g \in$ GA [\$/weight unit],

Average cost of manufacturing the group of items $g \in G A$ in the manufacturing plant $i \in P L$ [\$/weight unit],

Average cost of manufacturing the semi-finished group of items $e \in G A S$ in the plant $i \in P L$ [\$/weight unit], 
CACAP

$C_{\text {CPLPL }}$

$C_{\text {TPLCD }}{ }_{i j}$

$C T C D C D_{j n}$

${ }_{C T C D} A_{j k}$

$\mathrm{CTAA}_{\mathrm{kl}}$

$C T A Z_{k z}$

$\mathrm{CTCDZ}_{j z}$

$C F A_{k}$

$C F P L_{i}$

$C F C D_{j}$

CCIERRE $_{k}$

$C A P L_{i g}$

$C D E P L_{i g}$

$C C A P L_{i g}$

$C A C D_{j g}$

$C D E C D_{j g}$

$C C A C D_{j g}$

$C A A_{k g}$

$C D E A_{g k}$

$C C A A_{k g}$

$C A P C D_{j}$

CAPALM $_{k}$

CAPPR $_{\text {ig }}$

CAPPR $_{\text {ie }}$

factor $_{\text {eg }}$

$D E M A N D A_{g z}$

DEMANDADI $I_{g z}$
Cost of the increased production capacity for all group of items at plant $i \in P L$ [\$/weight unit],

Cost of transportation from plant $i \in P L$ to plant $q \in P L, i \neq q$ [\$/weight unit],

Cost of transportation from plant $i \in P L$ to distribution center $j \in C D$ [\$/weight unit],

Cost of transportation from distribution center $j \in C D$ to distribution center $n \in$ $C D, j \neq n$ [\$/weight unit],

Cost of transportation from distribution center $j \in C D$ to the warehouse $k \in A$ [\$/weight unit],

Cost of transportation from warehouse $k \in A$ to the warehouse $l \in A, k \neq l$ [\$/weight unit],

Cost of transportation from warehouse $k \in A$ to the market area $z \in Z$ [\$/weight unit],

Cost of transportation from distribution center $j \in C D$ to the market area $z \in Z$

[\$/weight unit],

Fixed cost of storage in the warehouse $k \in A$ [\$/ time unit],

Fixed cost of plant $i \in P L$ [\$/ time unit],

Fixed cost of distribution center $j \in C D[\$ /$ time unit],

Cost of closing of warehouse $k \in A$ [\$],

Set up cost of group of items $g \in \mathrm{GA}$ in the plant $\mathrm{i} \in \mathrm{PL}$ [\$/weight unit],

Cost of unloading the group of items $g \in G A$ in the plant $i \in P L$ [\$/weight unit],

Loading cost of the group of items $g \in G A$ in the plant $i \in P L$ [\$/weight unit],

Set up cost of group of the items $g \in G A$ in the distribution center $j \in C D$ [\$/weight unit],

Cost of unloading the group of items $g \in G A$ in the distribution center $j \in C D$ [\$/weight unit],

Cost of load of the group of items $g \in G A$ in the distribution center $j \in C D$ [\$/weight unit],

Set up cost of group of items $g \in G A$ in the warehouse $k \in A$ [\$/weight unit],

Cost of unloading the group of items $g \in G A$ in the warehouse $k \in A$ [\$/weight unit],

Loading cost of group of items $g \in G A$ in the warehouse $k \in A$ [\$/weight unit],

Capacity flow through the distribution center $j \in C D$ for all groups of items [weight unit / time unit],

Capacity flow of the warehouse $k \in A$ for all groups of items [weight unit / time unit],

Capacity production of the plant $i \in P L$ for the group of items $g \in G A$ [weight unit / time unit],

Capacity production of the plant $i \in P L$ for the group of items $e \in G A S$ [weight unit / time unit],

Percentage of raw material or semi-finished items $e \in G A S$ necessary to manufacture group of items $g \in G A 2$,

Demand of group of items $g \in G A$ in the market area $z \in Z$ which is attended from warehouses [weight unit / time unit]

Demand of group of items $g \in G A$ in the market area $z \in Z$ which is attended by direct delivery from distributions center [weight unit / time unit].

\section{Objective function}

The objective function of the MILP model is the maximization of EBITDA, represented as the difference between operating revenues and total costs excluding depreciation and amortization. 


\section{- Operating revenues}

Operating revenue is associated with the sales revenue generated by all groups of items in the market areas. Sales revenue from the warehouse and direct delivery (1) are considered:

$$
\sum_{g \in G A} \sum_{k \in A} \sum_{z \in Z} P V_{g z} \times F_{g k z}+\sum_{g \in G A} \sum_{j \in C D} \sum_{z \in Z} P V_{g Z} \times H_{g j z}
$$

Total costs include the sum of the costs associated with production in plants, additional capacity costs, costs of purchasing different groups of items, operating fixed costs, set up costs, costs unloading and loading, transportation costs and costs associated with the closing warehouses.

\section{- Production costs in plants}

These costs are associated with product manufacturing. This cost includes the values associated with finished and semi-finished items represented by Eq. (2):

$$
\sum_{i \in P L} \sum_{g \in G A} \sum_{j \in C D} C P_{g i} \times B_{g i j}+\sum_{g \in G A} \sum_{i \in P L} \sum_{e \in G A S} \sum_{q \in P L} C P G A S_{e i} \times P_{e i q g}
$$

\section{- Costs for additional production capacity}

Costs for additional production capacity arise when more storage capacity is needed in the production plants to meet demand of market areas. Costs associated with the finished goods and costs related to the raw materials or semi-finished items are considered:

$$
\sum_{i \in P L} \sum_{g \in G A} C_{A C A P_{i}} \times \text { Capdil }_{i g}+\sum_{i \in P L} \sum_{e \in G A S} C_{A C A P_{i}} \times \text { Capdil }_{i e}
$$

\section{- Purchasing costs of the group of items}

The company markets domestic products and other imported items. This cost considers the purchase of domestic as well as international products in the market areas and products sold by direct delivery:

$$
\sum_{g \in G A} \sum_{k \in A} \sum_{z \in Z} C C P_{g} \times F_{g k z}+\sum_{g \in G A} \sum_{j \in C D} \sum_{z \in Z} C C P_{g} \times H_{g j z}
$$

\section{- Operational Fixed costs of plants, distribution centers and warehouses}

These costs correspond to operational fixed costs of plants, distribution centers and warehouses are given as Eq. (5) as follows:

$$
\sum_{i \in P L} C F P L_{i}+\sum_{j \in C D} C F C D_{j}+\sum_{k \in A} C F A_{k} \times\left(1-W_{k}\right)
$$

\section{- Set up costs}

This category includes set up costs in plants, distribution centers and warehouses: 


$$
\begin{aligned}
& \sum_{i \in P L} \sum_{g \in G A} \sum_{j \in C D} C A P L_{i g} \times B_{g i j}+\sum_{i \in P L} \sum_{q \in P L} \sum_{g \in G A} \sum_{e \in G A S} C A P L_{i g} \times P_{e i q g} / i \neq q \\
& +\sum_{g \in G A} \sum_{j \in C D} \sum_{k \in A} C A C D_{j g} \times D_{g j k}+\sum_{g \in G A} \sum_{j \in C D} \sum_{n \in C D} C A C D_{j g} \times Y_{g j n} / j \neq n \\
& +\sum_{g \in G A} \sum_{z \in Z} \sum_{k \in A} C A A_{k g} \times F_{g k z}+\sum_{g \in G A} \sum_{k \in A} \sum_{l \in A} C A A_{k g} \times E_{g k l} / k \neq l
\end{aligned}
$$

\section{- Cost of unloading}

The costs of unloading in plants, distribution centers and warehouses are modeled in Eq. (7):

$$
\begin{aligned}
& \sum_{i \in P L} \sum_{q \in P L} \sum_{g \in G A} \sum_{e \in G A S} C D E P L_{q g} \times P_{e i q g} / i \\
& \neq q+\sum_{g \in G A} \sum_{n \in C D} \sum_{i \in P L} C D E C D_{n g} \times B_{g i n} \\
& +\sum_{g \in G A} \sum_{j \in C D} \sum_{n \in C D} C D E C D_{n g} \times Y_{g j n}+\sum_{g \in G A} \sum_{k \in A} \sum_{j \in C D} C D E A_{k g} \times D_{g j k} \\
& +\sum_{g \in G A} \sum_{k \in A} \sum_{l \in A} C D E A_{k g} \times E_{g k l} / l \neq k
\end{aligned}
$$

\section{- Cost of loading}

The costs of loading in plant, distribution centers and warehouses are modeled using Eq. (8):

$$
\begin{aligned}
& \sum_{i \in P L} \sum_{j \in C D} \sum_{g \in G A} C C A P L_{i g} \times B_{g i j}+\sum_{i \in P L} \sum_{q \in P L} \sum_{g \in G A} \sum_{e \in G A S} C C A P L_{i g} \times P_{e i q g} / i \neq q \\
& +\sum_{g \in G A} \sum_{j \in C D} \sum_{k \in A} C C A C D_{j g} \times D_{g j k}+\sum_{g \in G A} \sum_{j \in C D} \sum_{n \in C D} C C A C D_{j g} \times Y_{g j n} / j \neq n \\
& +\sum_{g \in G A} \sum_{j \in C D} \sum_{z \in Z} C C A C D_{j g} \times H_{g j z}+\sum_{g \in G A} \sum_{k \in A} \sum_{z \in Z} C C A A_{k g} \times F_{g k z} \\
& +\sum_{g \in G A} \sum_{k \in A} \sum_{l \in A} C C A A_{k g} \times E_{g k l} / k \neq l
\end{aligned}
$$

\section{- Transportation Costs}

In particular, the transportation costs between plants, plants and distribution centers, distribution centers and market areas, distribution center and warehouses, warehouses and between warehouses and market areas are modeled: 


$$
\begin{aligned}
& \sum_{g \in G A} \sum_{i \in P L} \sum_{e \in G A S} \sum_{q \in P L} C T P L P L_{i q} \times P_{e i q g}+\sum_{i \in P L} \sum_{g \in G A} \sum_{j \in C D} C T P L C D_{i j} \times B_{g i j} \\
& +\sum_{g \in G A} \sum_{j \in C D} \sum_{n \in C D} C T C D C D_{j n} \times Y_{g j n} / j \neq n+\sum_{g \in G A} \sum_{j \in C D} \sum_{z \in Z} C T C D Z_{j z} \times H_{g j z} \\
& \sum_{g \in G A} \sum_{j \in C D} \sum_{k \in A} C T C D A_{j k} \times D_{g j k}+\sum_{g \in G A} \sum_{k \in A} \sum_{l \in A} C T A A_{k l} \times E_{g k l} / k \neq l \\
& +\sum_{g \in G A} \sum_{k \in A} \sum_{z \in Z} C T A Z_{k z} \times F_{g k z}
\end{aligned}
$$

\section{- Warehouse Closing Costs}

The costs associated with the closure of warehouses are modeled by Eq. (10).

$$
\sum_{k \in A} \operatorname{CCIERRE}_{k} \times W_{k}
$$

\section{Constraints}

The constraints considered in the deterministic model are described below:

\section{- Production capacity constraints}

This set of constraints limiting the flow of items $g$ and semi-finished items $e$ sent from the plant $i$ to the plant $q$ or to distribution center $j$. This constraint is considered that some items $g$ can also be used as semi-finished products. Variables are added Capdil $_{i g}$ and Capdil2 $i e$ to capture extra capacity requirements. The groups of items that are not manufactured but are marketed are excluded (GACON and GACOI):

$$
\begin{aligned}
& \sum_{j \in C D} B_{g i j}+\sum_{q \in P L} \sum_{e \in G A S \mid e=g} \sum_{m \in G A P_{q} \mid m \in G A 2} P_{e i q m} \leq C A P P R_{i g}+\text { Capdil }_{i g} \\
& \forall g \in\{G A-\{G A C O N \cup G A C O I\}\} \\
& \forall i \in P L P_{g} \\
& \sum_{q \in P L} \sum_{m \in G A P_{q} \mid m \in G A 2} P_{e i q m} \leq C A P P R 2_{i e}+\text { Capdil }_{i e} \forall e \in G A S \mid e \neq g ; i \in \text { PLGAS }_{e}
\end{aligned}
$$

\section{- Balance constraints in plants}

These constraints are defined for all items $g$ that require manufacturing semi-finished items $e$. Eqs. (13) ensure that given the flow of items $B_{g i j}$, the flows necessary for manufacturing the items $g \in$ $G A 2\left(P_{\text {eqig }}\right)$, are determined.

$$
\sum_{q \in P L G A S_{e}} P_{\text {eqig }}=\text { factor }_{e g} \sum_{j \in C D} B_{g i j} \quad \forall g \in G A 2 ; \forall i \in P L P_{g} ; \forall e \in G A S G A_{g}
$$




\section{- Capacity constraints of distribution centers}

Capacity constraints of the distribution centers are associated with the flow of products handled. Using Eq. (14) is modeling the constraints in the input flow and with the Eq. (15) we determine the constraints in the outflow. This distinction is necessary because it is considered the entry to the network of sold items. Indeed, the output of products denoted by $D_{g j k}, Y_{g j n}$ and $H_{g j z}$ can be greater than the entry represented by $B_{g i j}$ and $Y_{g n j}$ :

$$
\begin{aligned}
& \sum_{i \in P L} \sum_{g \in G A} B_{g i j}+\sum_{g \in G A} \sum_{n \in C D \mid j \neq n} Y_{g n j} \leq C A P C D_{j} \quad \forall j \in C D \\
& \sum_{g \in G A} \sum_{k \in A} D_{g j k}+\sum_{g \in G A} \sum_{n \in C D \mid j \neq n} Y_{g j n}+\sum_{g \in G A} \sum_{z \in Z} H_{g j z} \leq C A P C D_{j} \quad \forall j \in C D
\end{aligned}
$$

\section{- Balance Constraints of distribution centers}

The balance equations for distribution centers are modeled by Eq. (16). The items that arrive at the distribution center should be the same products coming out. This equation excludes the sold items:

$$
\begin{array}{r}
\sum_{i \in P L \mid i \epsilon P L P_{g} \wedge i \in P L C D_{j}} B_{g j i}+\sum_{n \in C D \mid j \neq n \wedge n \epsilon C D G A_{g}} Y_{g n j} \\
=\sum_{k \in A} D_{g j k}+\sum_{n \in C D \mid j \neq n} Y_{g j n}+\sum_{z \in Z} H_{g j z}
\end{array}
$$

$\forall j \in C D ; \forall g \in\{G A-\{G A C O N \cup G A C O I\}\}$

\section{- Capacity constraints in warehouses}

Capacity constraints in the warehouses are associated with the flow of products, which handle distribution centers. In Eq. (17), the constraints on the input flow are modeled, and with Eqs. (18) the constraints of out flows are ensured:

$$
\begin{gathered}
\sum_{g \in G A} \sum_{j \in C D} D_{g j k}+\sum_{g \in G A} \sum_{l \in A \mid k \neq l} E_{g l k} \leq \text { CPALM }_{k} *\left(1-W_{k}\right) \quad \forall k \in A \\
\sum_{g \in G A} \sum_{z \in Z} F_{g k z}+\sum_{g \in G A} \sum_{l \in A \mid k \neq l} E_{g k l} \leq \text { CPALM }_{k} *\left(1-W_{k}\right) \quad \forall k \in A
\end{gathered}
$$

\section{- Balance equations of warehouses}

The balance constraints of warehouses are modeled by using Eqs. (19). Constraints (20) ensure the balance of flows of international items that only come to within distribution center $C D G A_{g}$ forg $\in$ GACOI:

$$
\sum_{j \in C D G A_{g}} D_{g j k}+\sum_{\substack{l \in A \mid k \neq l \\ \in A}} E_{g l k}=\sum_{z \in Z} F_{g k z}+\sum_{l \in A \mid k \neq l} E_{g k l} \forall g \in\{G A-\{G A C O N\}\}, \forall k
$$




$$
D_{g j k}=\sum_{z \in Z} F_{g k z}+\sum_{l \in A \mid k \neq l} E_{g k l} \forall g \in G A C O I, \forall j \epsilon C D G A_{g}, \forall k \in A
$$

\section{- Demand Constraints}

The demand of all customers must be satisfied by the warehouses. This constraint is satisfied by Eqs. (21) and the direct demand must be satisfied by the distribution centers given by Eqs. (22).

$\sum_{k \in A} F_{g k z}=D E M A N D A_{g z} \quad \forall g \in G A ; \forall z \in Z$

$\sum_{j \in C D G A_{g}} H_{g j z}=D E M A N D A I_{g z} \quad \forall g \in G A ; \forall z \in Z$

\section{- Non-negativity constraints}

$$
P_{g i q}, B_{g i j}, Y_{g j n}, D_{g j k}, E_{g k l}, F_{g k z}, H_{g j z}, \text { capdil }_{i g}, \operatorname{capdil}_{i e} \geq 0 ; \mathrm{Wk} \in\{0,1\}
$$

\subsection{Supply Network Design: Stochastic Model (SMILP)}

According to Kleywegt et al. (2002), the objective function for stochastic linear optimization problems can be formulated as follows,

$$
\min _{\mathrm{x} \in \mathrm{S}}\{g(x)=E[G(x, W)]\}
$$

In this case, $W$ corresponds to a random vector with an associated probability distribution $h$. $S$ is a finite set. $G(x, W)$ is a real function of two vector variables $x$ y $W$, and $E[G(x, W)]$ is its corresponding expected value. If we assume that the expected value function $g(x)$ is defined, therefore for each $x \in S$ the function $G(x, W)$ can be evaluated and the value is finite $E[G(x, W)]<\infty$. In order to formulate the stochastic model, it is necessary to consider the following formulation of the Stochastic Supply Network Design:

\section{Sets}

We have added the following set to the SMILP to those already declared for the MILP

$$
\text { ESCE Set of demand scenarios, indexed by } s=1,2, \ldots, N \text {. }
$$

\section{Operating parameters of SAA}

The parameters $D E M A N D A_{g z}$ and $D E M A N D A D I_{g z}$ has been removed for Deterministic Model by adding to the SMILP the following parameters:
N
Number of initial scenarios numbers to evaluate each solution of SMILP
$M$
Number of initial samples to generate Monte Carlo simulation (number of
$R$ times that solve the stochastic model for repetition of the SAA)
Counter of repetitions of SAA algorithm 
$C P 2_{g}$

$D E M A N D A_{g z s}$

$D E M A N D A D I_{g z s}$

$M E D E M A N D A_{g z s}$

$M E D E M A N D A D I_{g z s}$

DESDEMANDA $A_{g z S}$

DESDEMANDADI $I_{g z S}$
Average cost of manufacturing the group of items $g \in G A$ to penalize leftover and missing [\$/weight unit],

Demand for the group of items $g \in G A$ in the market area $z \in Z$ on the scenario $s \in E S C E$ [weight unit / time unit],

Demand for direct delivery of group of items $g \in G A$ in the market area $z \in$ $Z$ on the scenarios $s \in E S C E$ [weight unit / time unit].

Average of the demand for direct delivery of group of items $g \in G A$ in the market area $z \in Z$ on the scenarios $s \in E S C E$ [weight unit / time unit],

Average of the demand for direct delivery of group of items $g \in G A$ in the market area $z \in Z$ on the scenarios $s \in E S C E$ [weight unit / time unit],

Standard error of the demand for direct delivery of group of items $g \in G A$ in the market area $z \in Z$ on the scenarios $s \in E S C E$ [weight unit / time unit], Standard error of demand for direct delivery of group of items $g \in G A$ in the market area $z \in Z$ on the scenarios $s \in E S C E$ [weight unit / time unit]

\section{Operating Variables of SAA}

The following variables have been added to the variables already defined for the MILP.

\begin{tabular}{|c|c|}
\hline$F_{A L T A N T E S} S_{g z s}$ & $\begin{array}{l}\text { Unsatisfied quantities of group of items } g \in G A \text { in the market area } z \in \\
\text { respect to the demand of scenario } s \in E S C E \text { [weight unit / time unit], }\end{array}$ \\
\hline SOBRANTES $S_{g z s}$ & $\begin{array}{l}\text { Surplus of quantities of group of items } g \in G A \text { in the market area } z \in Z \text { respect } \\
\text { to the demand of scenario } s \in E S C E \text { [weight unit / time unit], }\end{array}$ \\
\hline FALTANTESDI $I_{g z S}$ & $\begin{array}{l}\text { Unsatisfied quantities of group of items } g \in G A \text { in the market area } z \in Z \\
\text { respect to the demand of direct deliveries of scenario } s \in E S C E \text { [weight unit / } \\
\text { time unit], }\end{array}$ \\
\hline SOBRANTESDI $I_{g z S}$ & $\begin{array}{l}\text { Surplus of quantities of group of items } g \in G A \text { in the market area } z \in Z \\
\text { respect to the demand of direct deliveries of scenario } s \in E S C E \text { [weight unit / } \\
\text { time unit], }\end{array}$ \\
\hline ventas $_{g z s}$ & $\begin{array}{l}\text { Sales of group of items } g \in G A \text { in the market area } z \in Z \text { respect to the demand } \\
\text { at scenario } s \in E S C E \text { [\$/time unit], }\end{array}$ \\
\hline ventasesp $_{g z}$ & Expected sales of group of items $g \in G A$ in the market area $z \in Z$ [\$/time unit], \\
\hline ven & $\begin{array}{l}Z \text { respect to the demand } \\
\text { init], }\end{array}$ \\
\hline ventasdiesp $p_{g z}$ & $\begin{array}{l}\text { Expected sales of group of items } g \in G A \text { in the market area } z \in Z \text { by direct } \\
\text { deliveries [\$/time unit], }\end{array}$ \\
\hline COSTPENSOBFALT & $\begin{array}{l}\text { Variable equal to the total cost of penalization by unsatisfied demand or } \\
\text { surplus of demand of the group of items } g \in G A \text { in the market area } z \in Z \\
\text { respect to the demand and direct demand for all scenarios } s \in E S C E \text { [\$/time } \\
\text { unit], }\end{array}$ \\
\hline FO & $\begin{array}{l}\text { Variable equal to the value of the real objective function, i.e. without the } \\
\text { penalization cost, }\end{array}$ \\
\hline $\operatorname{cosec} s$ & $\begin{array}{l}\text { Variable equal to the value of the objective function with the penalization } \\
\text { scheme for each demand scenario } s \in E S C E \text {. }\end{array}$ \\
\hline
\end{tabular}

\section{Objective function with the penalization cost}

In the objective function of the SMILP, the equation (1) has been replaced by Eqs. (25) and Eq. (26) has been added to the objective function (1) - (10). Eqs. (26) represent the penalization cost caused by the configuration of the supply network after the variability of the demand. 
$\sum_{g \in G A} \sum_{z \in Z} P V_{g z} \times$ ventasesp $_{g z}+\sum_{g \in G A} \sum_{Z \in Z} P V_{g z} \times$ ventasdiesp $_{g z}$

- COSTPENSOBFALT

$$
\begin{aligned}
& =\left(\frac{1}{N}\right)\left[\sum_{g \in G A} \sum_{z \in Z} \sum_{s \in E S C E} C P 2_{g} \times\left(\text { FALTANTES }_{g z s}+\text { FALTANTESDI }_{g z s}\right)\right. \\
& \left.+\sum_{g \in G A} \sum_{z \in Z} \sum_{s \in E S C E}\left(C P 2_{g}\right) \times\left(\text { SOBRANTES } S_{g z s}+\text { SOBRANTESDI }{ }_{g z s}\right)\right]
\end{aligned}
$$

The Eq. (26) determines the total expected penalty cost by unsatisfied demand or surplus of demand that has been included in the objective function.

\section{Constraints of the SMILP}

Demand constraints of the deterministic model represented in equation (21) and (22) have been eliminated by adding the following constraints in the stochastic model structure.

\section{- Unsatisfied demand and surplus of demand}

The determination of the unsatisfied demand and surplus of demand respect to the demand and direct demand are given by Eqs. (27) and Eqs. (28) respectively.

$$
\begin{aligned}
& \text { FALTANTES }_{g z s}-\text { SOBRANTES } S_{g z s}=D E M A N D A_{g z s}-\sum_{k \in A} F_{g k z} \forall g \in G A ; z \in Z ; s \\
& \quad \in E S C E \\
& \text { FALTANTESDI }_{g z s}-S O B R A N T E S D I_{g z s}=D E M A N D A D I_{g z s}-\sum_{j \in C D G A_{g}} H_{g j z} \forall g \in G A ; z \in Z ;
\end{aligned}
$$

$s \in E S C E$

\section{- Sales}

The sales generated by group of item quantities sent to a market area and the variability of the demand respect the demand is shown in Eq. (30) and Eq. (31) respectively.

$$
\begin{aligned}
& \text { SOBRANTES } \text { gzs }=\sum_{k \in A} F_{g k z}-\text { ventas }_{g z s} \forall g \in G A ; \forall z \in Z ; \forall s \in E S C E \\
& \text { SOBRANTESDI } I_{g z s}=\sum_{j \in C D G A_{g}} H_{g j z}-\text { ventas }_{g z s} \forall g \in G A ; \forall z \in Z ; \forall S \in E S C E
\end{aligned}
$$

\section{- Expected sales}

Expected sales are modeled Eq. (32), which represents sales by demand, and Eq. (33) represents sales by direct demand.

$$
\begin{aligned}
& \text { ventasesp }_{\mathrm{gz}}=\sum_{\mathrm{s} \in \mathrm{ESCE}}\left(\frac{1}{\mathrm{~N}}\right) \text { ventas }_{\mathrm{gzs}} \quad \forall \mathrm{g} \in \mathrm{GA} ; \forall \mathrm{z} \in \mathrm{Z} \\
& \text { ventasdiesp }_{g \mathrm{z}}=\sum_{s \in E S C E}\left(\frac{1}{N}\right) \text { ventasdi }_{g z s} \quad \forall g \in G A ; \forall z \in Z
\end{aligned}
$$




\section{- Additional constraints related to the objective function of the SMILP}

The constraints equivalent to the objective function of MILP has been added to the SMILP. Revenues are the expected value of sales (Eq. (1) is replaced with (25); penalization by unsatisfied demand or surplus of demand is excluded).

$$
F O=\text { Eqs. (25) }- \text { [Eqs. (2) }-(10)]
$$

Similarly, the objective function has been determined to evaluate at each scenario $s \in E S C E$ as Eq.

(35)

$$
\begin{aligned}
& \text { FOesce }_{s}=\sum_{g \in G A} \sum_{z \in Z} \sum_{s \in E S C E}\left(P V_{g z} * V E N T A S_{g z s}\right)+\sum_{g \in G A} \sum_{z \in Z} \sum_{s \in E S C E}\left(P V_{g z} * V E N T A S D I_{g z s}\right) \\
& \text { - [Eqs. (2) - (10)] } \forall s \in E S C E
\end{aligned}
$$

\section{Solution strategy SAA for SMILP}

According to Santoso et al. (2005), the SAA algorithm is summarized in 4 steps:

1. Generate $M$ independent samples of size $N$ each, for $j=1, \ldots, M$. For each sample solve the corresponding SAA problem.

$$
\min _{w \in W}\left\{c^{T} w+\frac{1}{N} \sum_{n=1}^{N} Q\left(w, \xi_{j}^{n}\right)\right\}
$$

For each $j$, it is possible to obtain the optimum value and the corresponding optimal solution, $v_{\mathrm{N}}$ and $\hat{y}_{\mathrm{N}}$.

2. Calculate the next statistical indicators:

$$
\begin{aligned}
& \bar{v}_{N, M}=\frac{1}{M} \sum_{j=1}^{M} v_{N}^{j} \\
& \sigma_{\bar{v}, N, M}^{2}=\frac{1}{(M-1) M} \sum_{j=1}^{M}\left(v_{N}^{j}-\bar{v}_{N, M}\right)^{2}
\end{aligned}
$$

The value of $\bar{v}_{N, M}$ provides a statistical lower bound of the real optimal $(v *)$ of the original problem, while Eq. (38) is a estimator of its variance.

3. Select a feasible solution $\bar{w} \in W$ of the real problem, using as example the best of solutions $\widehat{w}_{\mathrm{N}}^{j}$ calculated in (64). Calculate the objective function value off( $(\bar{w})$, using Eq. (39).

$$
\tilde{f}_{N^{\prime}}(\bar{w})=c \bar{w}+\frac{1}{N^{\prime}} \sum_{n=1}^{N^{\prime}} Q\left(\bar{w}, \xi^{n}\right)
$$

In the above equation, $\left(\xi_{j}^{1}, \ldots ., \xi_{j}^{N}\right)$ is an sample of size $N^{\prime}$ which is independent of the samples used in step 1. In general, it is usual to take a value $N^{\prime}$ much greater than $N$. Since the samples are independent and identically distributed, the variance of Eq. (39) can be expressed as follows:

$$
\sigma_{N^{\prime}}^{2}(\bar{w})=\frac{1}{\left(N^{\prime}-1\right) N} \sum_{j=1}^{N^{\prime}}\left[c \bar{w}+Q\left(\bar{w}, \xi^{n}\right)-\hat{f}_{N^{\prime}}(\bar{w})\right]
$$

In this case, since the problem being solved is maximization, it is natural to choose $\bar{w}$ with the highest estimated objective function value $\widetilde{f}_{N^{\prime}}(\bar{w})$. 
4. Calculate an estimate of the optimality gap of solution $\overline{\mathrm{w}}$ by using the results obtained in steps 2 and 3 as follows:

$$
\operatorname{gap}_{N, M, N}\left(\overline{w)}=\tilde{f}_{N^{\prime}}(\bar{w})-\bar{v}_{N, M}\right.
$$

The estimated gap variance is calculated as follows:

$$
\sigma_{g a p}^{2}=\sigma_{N^{\prime}}^{2}(\bar{w})+\sigma_{\bar{v}, N, M}^{2}
$$

The SAA algorithm developed in this study is based on the steps proposed by Santoso et al. (2005). This optimization algorithm must to solve initially a sample of $M$ problems of the deterministic model each one with $N$ demand scenarios generated by Monte Carlo Simulation. The indicators of the SAA is calculated for the supply network design for $N^{\prime}=3 N$. If the stop criteria $(g a p \leq 5 \%)$ or $(R=50)$ are not met, the algorithm is repeated with $M=2 M$ until find an optimal solution.

\section{Computational results}

\subsection{Characteristics of the real case company}

The deterministic (MILP) and the stochastic model (SMILP) have been validated for a real case taken from a Colombian company, which manufactures product for the bakery industry. The industry under study is a company with a history of over 60 years in the Colombian industrial sector, with sales revenue of over 430 billion of pesos per year and a budget of 50 billions of pesos per year to implement logistics operations. The supply network of the company consists of 6 factories, 6 distribution centers, 15 warehouses and 15 commercial districts with storage capacity. In addition, the company has a fleet consisting of 230 vehicles and more than 3000 customers. There is a continuous flow of product by trucks among production plants, distribution centers and warehouses. Several suppliers provide the marketed products, which are located in different echelons at the network. Imported products for marketing arrive to distribution centers, while the national products are provided by suppliers directly to the warehouses. Distribution centers serve the customers by direct delivery the demand of products for some special customers and the demand for 15 commercial areas distributed throughout the country. We want to know whether or not the number and location of warehouses is optimal.

\subsection{Obtained results}

The optimization models (deterministic and stochastic) and the SAA strategy have been implemented on $\mathrm{C}++$, and the experiments have been executed in an Intel Core i7 processor with OS Windows 7 and memory of 8 GB. CPLEX 12.5 has been used to solve MILP and SMILP.

\subsection{Deterministic Model (MILP)}

The results obtained by the deterministic model (MILP) are shown in Table 1. Column 2 indicates the objective function value (EBITDA). Column 3 indicates the warehouses to be closed for optimum network design.

\section{Table 1}

Objective function values (EBITDA) and supply network configuration (Deterministic Model)

\section{$\begin{array}{lll}\text { Scenario } & \text { EBITDA } & \text { Closed Warehouses }\end{array}$}

\begin{tabular}{lll}
\hline Current situation & $\$ 80.613 .788 .040$ & \\
Deterministic Model & $\$ 82.406 .831 .500$ & $4,6,8,12$ y 13 \\
\hline
\end{tabular}


According to the results shown in Table 1, it is necessary to operate with 11 warehouses. Indeed, warehouses 4, 6, 8, 12 and 13 must be closed. An improvement of the objective function of approximately 1700 million of pesos is obtained respect to the current situation of the company.

\subsection{Stochastic Model (SMILP)}

For the execution of the SAA methodology, 6 different sample sizes were used. The sample sizes considered are: $N=5$ and $M=5 ; N=10$ and $M=10 ; N=20$ and $M=20 ; N=30$ and $M=30$; $N=40, M=40$; and $N=50, M=50$.

Fig. 1 represents the behavior of value $\bar{v}_{N, M}$ and $\tilde{f}_{N^{\prime}}(\bar{w})$. In this way the convergence of the proposed method by Kleywegt et al. (2002) is validated.

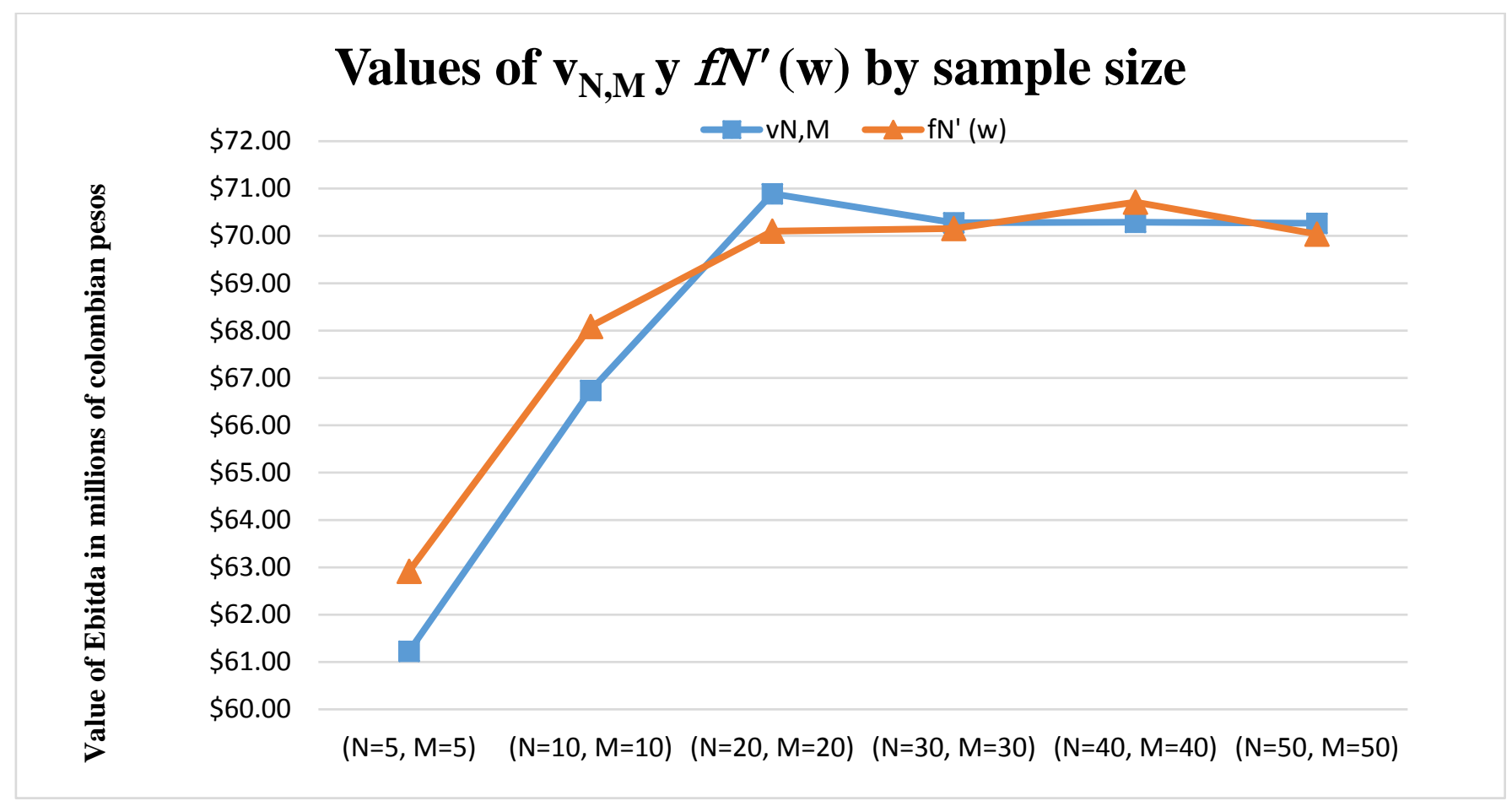

Fig. 1. Values of $\bar{v}_{N, M} \mathrm{y} \tilde{f}_{N^{\prime}}(\bar{w})$ by sample size

Table 2 shows the results obtained applying the SAA procedure for different values of $N$ and $M$. The columns of the Table 2 show the SAA indicators. According to these results, the best value of the obtained objective function is $\$ 70713^{\prime} 451.516,5$, which is associated with a gap of $0.60 \%$. For this reason, it is selected as the optimal configuration associated with this objective function value.

Table 3 compares the results obtained by the deterministic (MILP) and the stochastic model (SMILP). Note that the difference of results between two models confirming that the lack of consideration of the variability in demand, could lead to wrong decisions on the supply chain configuration. The objective function value of the stochastic model (SMILP) is smaller than those value reported for the deterministic model (MILP), because the operating revenues are greater in the MILP. While the demand of the deterministic model is fully satisfied, the demand of the stochastic model is not completely satisfied as consequence of uncertainty of demand. In this way, is possible to have unsatisfied or surplus of demand. 
Table 2

Result Report by SAA procedure

\begin{tabular}{ccccccc}
\hline$N, M, N^{\prime}$ & $\bar{v}_{N, M}$ & $\sigma_{\bar{v}, N, M}^{2}$ & $\tilde{f}_{N^{\prime}}(\bar{w})$ & $\sigma_{N^{\prime}}^{2}(\bar{w})$ & $g a p_{N, M, N}(\bar{w})$ & $\sigma_{g a p}^{2}$ \\
\hline $5,5,15$ & $\$ 61,226,107,620.79$ & $\$ 427,478,308.61$ & $\$ 62,918,328,601.69$ & $\$ 753,495,515.86$ & $2.69 \%$ & $\$ 866,310,104.27$ \\
$10,10,30$ & $\$ 66,734,370,354.78$ & $\$ 198,540,231.76$ & $\$ 68,088,733,143.98$ & $\$ 660,179,593.98$ & $1.99 \%$ & $\$ 689,387,641.27$ \\
$20,20,60$ & $\$ 70,890,309,060.96$ & $\$ 199,151,076.57$ & $\$ 70,100,155,058.12$ & $\$ 679,213,648.53$ & $1.13 \%$ & $\$ 707,808,117.82$ \\
$30,30,90$ & $\$ 70,276,717,102.29$ & $\$ 118,882,236.05$ & $\$ 70,156,823,241.09$ & $\$ 594,696,059.93$ & $0.17 \%$ & $\$ 606,462,191.52$ \\
$40,40,120$ & $\$ 70,288,720,319.36$ & $\$ 88,434,482.36$ & $\$ 70,713,451,516.50$ & $\$ 474,008,019.44$ & $0.60 \%$ & $\$ 482,186,955.62$ \\
$50,50,150$ & $\$ 70,265,362,082.05$ & $\$ 73,207,481.53$ & $\$ 70,038,504,705.21$ & $\$ 552,177,329.79$ & $0.32 \%$ & $\$ 557,009,101.26$ \\
\hline
\end{tabular}

Table 3

Comparison of deterministic and stochastic model.

\begin{tabular}{cccc}
\hline Model & EBITDA & Open Warehouses & Closed Warehouses \\
\hline Deterministic (MILP) & $\$ 82.406 .831 .500$ & $1,2,3,4,5,6,7,8,9,10,11,12,13,14,15$ & $4,6,8,12,13$ \\
Stochastic (SMILP) & $\$ 70.713 .451 .516$ & $1,2,3,5,10,11,14,15$ & $4,6,7,8,9,12,13$ \\
\hline
\end{tabular}

\section{Conclusion}

This paper has considered the redesign of a distribution network for a large-scale consumer products company by considering aspects of deterministic demand through Mixed Integer Linear Programming Deterministic Models and stochastic aspects with Mixed Integer Linear Programming Stochastic Models. The proposed stochastic model has determined the strategic and tactical decisions in one stage, presenting a novel approach to linear stochastic problems solution. The algorithmic strategy SAA has been used to solve the stochastic model, which uses an approximation scheme for sample averages for solving stochastic problems. We have compared the configuration of logistics warehouses for both cases (deterministic and stochastic models), confirming the importance of the consideration of variability of the demand in the Supply Network Design (SND).The results show that the proposed methodology was a solid proposal to support decisions of SND by considering the expected economic contribution of products and variability of the demand. In addition, we have demonstrated that the methodology SAA provides near-optimal solutions for linear stochastic programming problems with small sample sizes. Dynamic aspects of the network, which help to make decisions according to the demand seasonality, could be considered as future research work. In addition, decisions of transportation modes, and test of efficiency and applicability of other methods for solving stochastic linear optimization models could be considered as extensions of this paper.

\section{References}

Arabani, A. B., \& Farahani, R. Z. (2012). Facility location dynamics: An overview of classifications and applications. Computers \& Industrial Engineering, 62(1), 408-420.

Ballou, R. (2004). Busssines logistics management: Planning, organizing and controlling the supply chain. fifth edition, Prentice Hall, Upper Saddle River, New Jersey.

Beamon, B. M. (1999). Measuring supply chain performance. International Journal of Operations \& Production Management, 19(3), 275-292.

Berman, O., \& Drezner, Z. (2003). A probabilistic one-centre location problem on a network. Journal of the Operational Research Society, 54, $871-877$.

Chaudhry, S. S., \& Luo, W. (2005). Application of genetic algorithms in production and operations management: a review. International Journal of Production Research, 43(19), 4083-4101.

Chen, C. L., Wang, B. W., \& Lee, W. C. (2003). Multiobjective optimization for a multienterprise supply chain network. Industrial \& Engineering Chemistry Research, 42(9), 1879-1889. 
Chen, G., Daskin, M. S., Shen, Z. J., \& Uryasev, S. (2005). A new model for stochastic facility location modeling. Research Report, Department of Industrial and Systems Engineering, University of Florida.

Chouinard, M., D’Amours, S., \& Aït-Kadi, D. (2008). A stochastic programming approach for designing supply loops. International Journal of Production Economics, 113(2), 657-677.

Coronado-Hernández, J. R., García-Sabater, J. P., Maheut, J., \& Garcia-Sabater, J. J. (2010, October). Modelo de optimización estocástica para la planificación de cadenas de suministro para productos con ciclo de vida cortos. In 4th International Conference On Industrial Engineering and Industrial Management (pp. 1366-1375).

Current, J., Ratick, S., \& ReVelle, C. (1998). Dynamic facility location when the total number of facilities is uncertain: A decision analysis approach. European Journal of Operational Research, 110(3), 597609.

Dantzig, G. B. (1955). Linear programming under uncertainty. Management Science, 1(3), 197-206.

Escobar J.W. (2012). Rediseño de una red de distribución con variabilidad de demanda usando la metodología de escenarios. Revista Facultad de Ingeniería UPTC, 21(32), 9-19.

Escobar J.W., Bravo, J.J., \& Vidal, C.J. (2012). Optimización de redes de distribución de productos de consumo masivo en condiciones de riesgo, Proceedings of XXXIII Congreso Nacional de Estadística e Investigación Operativa (SEIO), Madrid, Spain.

Escobar, J. W. (2009). Modelación y Optimización de diseño de redes de distribución de productos de consumo masivo con elementos estocásticos. Proceedings of XIV Latin American Summer Workshop on Operations Research (ELAVIO), El Fuerte, México.

Escobar, J. W., Bravo, J. J., \&Vidal, C. J. (2013). Optimización de una red de distribución con parámetros estocásticos usando la metodología de aproximación por promedios muéstrales. Ingeniería y Desarrollo, 31(1), $135-160$.

Gabor, A. F., \& Van Ommeren, J. C. W. (2006). An approximation algorithm for a facility location problem with stochastic demands and inventories. Operations research letters, 34(3), 257-263.

Kleywegt, A. J., Shapiro, A., \& Homem-de-Mello, T. (2002). The sample average approximation method for stochastic discrete optimization. SIAM Journal on Optimization, 12(2), 479-502.

Klose, A., \& Drexl, A. (2005). Facility location models for distribution system design. European Journal of Operational Research, 162(1), 4-29.

Lieckens, K., \& Vandaele, N. (2007). Reverse logistics network design with stochastic lead times. Computers \& Operations Research, 34(2), 395-416.

Listeş, O., \& Dekker, R. (2005). A stochastic approach to a case study for product recovery network design. European Journal of Operational Research,160(1), 268-287.

Meixell, M. J., \& Gargeya, V. B. (2005). Global supply chain design: A literature review and critique. Transportation Research Part E: Logistics and Transportation Review, 41(6), 531-550.

Melo, M. T., Nickel, S., \& Saldanha-Da-Gama, F. (2009). Facility location and supply chain management-A review. European Journal of Operational Research, 196(2), 401-412.

Owen, S. H., \& Daskin, M. S. (1998). Strategic facility location: A review.European Journal of Operational Research, 111(3), 423-447.

Petrovic, D., Xie, Y., Burnham, K., \& Petrovic, R. (2008). Coordinated control of distribution supply chains in the presence of fuzzy customer demand. European Journal of Operational Research, 185(1), 146-158.

Schütz, P., Tomasgard, A., \& Ahmed, S. (2009). Supply chain design under uncertainty using sample average approximation and dual decomposition. European Journal of Operational Research, 199(2), 409-419.

Shen, Z. (2007). Integrated supply chain design models: a survey and future research directions. Journal of Industrial and Management Optimization, 3(1), 1.

Snyder, L. V. (2006). Facility location under uncertainty: a review. IIE Transactions, 38(7), 547-564. 